bioRxiv preprint doi: https://doi.org/10.1101/2021.02.17.431616; this version posted May 11, 2021. The copyright holder for this preprint (which was not certified by peer review) is the author/funder, who has granted bioRxiv a license to display the preprint in perpetuity. It is made available under aCC-BY-NC-ND 4.0 International license.

\title{
Spatiotemporal organization of chromatin domains: role of interaction energy and polymer entropy
}

\author{
Kiran Kumari, ${ }^{1,2,3}$ J. Ravi Prakash, ${ }^{3}$ and Ranjith Padinhateeri ${ }^{2}$ \\ ${ }^{1}$ IITB-Monash Research Academy, Indian Institute of Technology Bombay, Mumbai, Maharashtra - 400076, India \\ ${ }^{2}$ Department of Biosciences and Bioengineering, Indian Institute of Technology Bombay, Mumbai 400076, India \\ ${ }^{3}$ Department of Chemical Engineering, Monash University, Melbourne, VIC 3800, Australia
}

\begin{abstract}
Chromatin is known to be organised into multiple domains of varying sizes and compaction. While these domains are often imagined as static structures, they are highly dynamic and show cell-to-cell variability. Since processes such as gene regulation and DNA replication occur in the context of these domains, it is important to understand their organization, fluctuation and dynamics. To simulate chromatin domains, one requires knowledge of interaction strengths among chromatin segments. Here, we derive interaction strength parameters from experimentally known contact maps, and use it to predict chromatin organization and dynamics. Taking $\alpha$-globin domain as an example, we investigate its 3D organization, size/shape fluctuations, and dynamics of different segments within a domain, accounting for hydrodynamic effects. Perturbing the interaction strengths systematically, we quantify how epigenetic changes can alter the spatio-temporal nature of the domains. Computing distance-distributions and relaxation times for different chromatin states, we show that weak and strong interactions cooperatively determine the organization of the domains and how the solid-like and liquid-like nature of chromatin gets altered as we vary epigenetic states. Quantifying dynamics of chromatin segments within a domain, we show how the competition between polymer entropy and interaction energy influence the timescales of loop formation and maintenance of stable loops.
\end{abstract}

PACS numbers:

\section{INTRODUCTION}

Chromatin is a long polymer made of DNA and proteins; its four dimensional (spatial and temporal) organization is crucial in regulating cellular processes like transcription, replication and DNA repair [1-4]. How the chromatin self-organises spatially and temporally is a question of active research. Recent developments in chromosome conformation capture (Hi-C) [4-9] and microscopy experiments [10-12] help us investigate contacts among different segments and overall 3D organization of the chromatin polymer. Such experiments so far suggest that intra-chromatin interactions lead to the formation of different types of domains within a single chromosome $[6,7,11-15]$.

It has been hypothesized that loop extrusion and/or phase separation could be the mechanism for the formation of these domains $[14,16,17]$. In the loop extrusion picture, chromatin regions are actively brought together with the help of proteins like cohesins/codensins and held together by CCCTC-binding factor (CTCF) $[13,14,18$, 19]. However, CTCF-dependent loops are found only in a fraction of the domains $[13,20]$. Hence an alternative proposal is that chromatin domains may also be passively formed via phase separation $[17,21,22]$. Recently, it has been shown that in the absence of loop extruding factors, chromatin does still form domains and execute the necessary biological function [11, 17, 20, 23, 24] indicating that micro phase-separation might be an important mechanism. Phase separation is also known to bring together certain enhancers and promoters segregating them from other regions $[21,22,25]$. In certain cases, as far as biological function is concerned, there is an ongoing debate whether actual contact is crucial or proximity closeness in 3D without being physically in contact would suffice $[3,17,23,26]$. To probe this further, it is important to go beyond contacts and examine the whole configurational space of chromatin polymer.

Widely used experimental methods so far provide us only static snapshots of chromatin contacts. Large Hi-C contact values are often assumed to represent static contacts holding together different segments of chromatin. However, note that experimentally inferred contact probability values, for nearly all chromatin segments, are very small $(<<1)$ [13]. This implies that the contacts will be often broken and the chromatin polymer conformation can be highly dynamic. Even in a steady-state, there are likely to be large fluctuations, cell to cell and temporal variabilities. While there have been several attempts to understand the static 3D configurations and their average properties, the dynamic nature of chromatin domains remains unclear.

Complementing recent experimental efforts, there have been several computational studies investigating chromatin organization. [18, 19, 27-42]. Many studies carried out "forward" simulations where the chromatin structure is predicted assuming a set of interaction strengths [42]. However, we do not know the optimal values of interaction strengths a priori. One requires an inverse method that can obtain the optimal interaction strengths such that experimentally known properties are recovered. We have recently developed an Inverse Brownian Dynamics (IBD) method to extract potential energy parameters that are consistent with HiC-like experiments [29]. The 
bioRxiv preprint doi: https://doi.org/10.1101/2021.02.17.431616; this version posted May 11, 2021. The copyright holder for this preprint (which was not certified by peer review) is the author/funder, who has granted bioRxiv a license to display the preprint in perpetuity. It is made available under aCC-BY-NC-ND 4.0 International license.

method explicitly computes intra-chromatin interactions for every segment pair. Using the intra-chromatin interactions computed from this method, in this work we go beyond the static picture and study chromatin fluctuations and dynamics perturbing epigenetic states. We use Brownian dynamics (BD) simulations with hydrodynamic interactions for this study. Going beyond contact frequencies, we compute the full distance distribution between all pairs of segments and examine the cooperative nature of folding. We then study the dynamics of the domain, compute relaxation times, loop formation times and contact times. We investigate how the interaction strengths and polymer entropy influence these measurable quantities. Finally, we discuss the significance of these findings.

\section{MODEL AND METHODS}

We consider chromatin as a bead spring chain having optimal intra-chromatin interactions derived from $5 \mathrm{C}$ data using an Inverse Brownian Dynamics (IBD) algorithm [29]. The total energy of the chromatin bead spring chain, made of $N$ beads, is $U=U^{\mathrm{S}}+U^{\mathrm{SDK}}$ where $U^{\mathrm{S}}=\sum_{i} \frac{H}{2}\left(\left|\boldsymbol{r}_{i}-\boldsymbol{r}_{i+1}\right|-r_{0}\right)^{2}$ is the spring potential between the adjacent beads $i$ and $(i+1), \boldsymbol{r}_{i}$ is the position vector of bead $i, r_{0}$ is the natural length and $H$ is the stiffness of the spring [43]. To mimic protein-mediated interactions between bead-pairs, we use Soddemann-Duenweg-Kremer $\left(U^{\mathrm{SDK}}\right)$ potential whose repulsive part is modelled by Weeks-Chandler-Anderson potential $4\left[\left(\frac{\sigma}{r_{i j}}\right)^{12}-\left(\frac{\sigma}{r_{i j}}\right)^{6}+\frac{1}{4}\right]-\epsilon_{i j}$ upto the range $2^{1 / 6} \sigma$. Here $\sigma$ is the parameter that determines the minima of the potential, $r_{i j}=\left|\boldsymbol{r}_{i}-\boldsymbol{r}_{j}\right|$ is the distance and $\epsilon_{i j}$ is an independent parameter representing the attractive interaction strength between beads $i$ and $j$. The repulsive part is unaffected by the choice of potential parameter $\epsilon_{i j}$. The attractive part is modelled by $\frac{1}{2} \epsilon_{i j}\left[\cos \left(\alpha r_{i j}^{2}+\beta\right)-1\right]$ which, unlike the LennardJones potential, smoothly reaches zero at the cut off radius $r_{c}=1.82 \sigma[44,45]$. Parameters $\alpha$ and $\beta$ control the $r_{c}$ (see supplementary information (SI)). We simulate the chromatin polymer using Brownian dynamics simulations, where the time evolution of the bead positions are governed by an Itô stochastic differential equation. This simulation accounts for hydrodynamics interaction which is computed by the regularized Rotne-Prager-Yamakawa tensor. See SI for details. For the simulation, all the length and time scales are non-dimensionalised with $l_{H}=\sqrt{k_{\mathrm{B}} T / H}$ and $\lambda_{H}=\zeta / 4 H$, respectively where $T$ is the absolute temperature, $k_{\mathrm{B}}$ is the Boltzmann constant, and $\zeta=6 \pi \eta_{s} a$ is the Stokes friction coefficient of a spherical bead of radius $a$ with $\eta_{s}$ being the solvent viscosity. All the non-dimensional quantities are indicated with the asterik $(*)$ symbol such as non-dimensional po- sition $r_{i}^{*}=r_{i} / l_{H}$.

For a chromatin, we do not know the interaction energy strengths à priori. Given $5 \mathrm{C} / \mathrm{HiC}$ data, we performed an inverse calculation and obtained the optimal interaction strengths that are consistent with the experimental contact probability map [29]. The inverse algorithm works as follows: we start with the initial guess values of interaction strengths, simulate the polymer following the conventional forward Brownian dynamics method and obtain the simulated contact probabilities in the steady state. Based on a statistical method, the interaction strengths are revised for the next iteration, depending upon the difference between the simulated and the known experimental contact probabilities (see SI). We perform several iterations of the loop (i.e., BD simulation, calculation of contact probability and revision of interaction strength) until the error between the simulated and experimental contact probabilities is less than a predetermined tolerance value.

\section{RESULTS AND DISCUSSION}

As a first step, to establish a connection between our simulations and experiments, we computed the average 3D spatial distance $\left(r_{i j}^{*}=\left|\mathbf{r}_{i}^{*}-\mathbf{r}_{j}^{*}\right|\right)$ between different pairs of chromatin segments as a function of the corresponding 1D genomic separation $\left(s_{i j}=|i-j|\right)$. As shown in Fig. 1(a), $\left\langle r_{i j}^{*}\right\rangle \sim s_{i j}^{\nu}$ with a scaling exponent $\nu=0.38$ in the OFF state, suggesting near close packing within the chromatin domain (red symbols). As a "control", we also simulated a self-avoiding walk (SAW) polymer with no attractive interaction $\left(\epsilon_{i j}=0\right)$ which results in $\nu=0.6$ as expected (black symbols) [43]. Our chromatin model predicts a spread (variability) in the $3 \mathrm{D}$ distance (red symbols) which is absent in the control revealing the implications of heterogeneous intra-chromatin interactions. A recent microscopy study [12] on a mouse ESC chromatin domain also showed a similar behavior - both the scaling (slope) and the variability in the experimental and simulation data are comparable without any fitting parameter. This is a validation that macroscopic polymer properties of chromatin domain in our simulation accurately represent what is observed in realistic systems. The $y$-intercept of the experimental data gives us the size $\left(\sigma=l_{H}\right)$ of the $10 \mathrm{~kb}$ chromatin (a single bead in our simulation). For this experimental system (mouse chromosome 6, 1.2MB in Szabo et al. [12]) we get $l_{H}=22 \mathrm{~nm}$. Even though we do not have such extensive spatial distance data for $\alpha$ globin, we compared the available FISH data for $\alpha$ globin and deduced the $l_{H}=36 \mathrm{~nm}$ (see SI). Throughout this paper, $l_{H}=36 \mathrm{~nm}$ and $\lambda_{H}=0.1 \mathrm{~s}$ are used to convert all non-dimensional lengths and times into standard units and we will present quantities in both units. The reasons for the choice of both these specific values are discussed in greater detail in the SI. 
bioRxiv preprint doi: https://doi.org/10.1101/2021.02.17.431616; this version posted May 11, 2021. The copyright holder for this preprint

\section{Distance distributions and cooperative nature of chromatin folding}

Even though the average distance between two chromatin segments is often used to represent chromatin organization, this may not describe the accurate biological picture in a dynamic, heterogeneous context. We compute the distribution of $3 \mathrm{D}$ distance between different segments, $p\left(r^{*}\right)$, as it captures the maximum information about variability and fluctuations of chromatin. As a control, we computed the $p\left(r^{*}\right)$ for a SAW and it agrees well with the known analytical expression of des Cloizeaux, $p\left(\boldsymbol{r}^{*}\right)=C\left(r^{*}\right)^{\theta+2} e^{-\left(K r^{*}\right)^{1 /(1-\nu)}}[46]$. Here $\nu$ is the Flory exponent, $\theta$ is a geometrical exponent and the coefficients $C$ and $K$ are functions of $\theta$ [46]. Recent work has led to an accurate estimation for these constants which are discussed in SI. Fig. 1(b) shows the validation with intermediate beads of a SAW (with $\nu=0.6, \theta=0.81, K=1.17, C=2.05)$.

We then studied the $p\left(r^{*}\right)$ for the $\alpha$-globin gene locus in GM12878 cell type. Examining various segments $250 \mathrm{~kb}$ apart along the chain backbone (25 beads), we find that all the distributions have a broad peak near their respective average distances (Fig. 1(c)). However, for bead pairs having high $\epsilon$ values, a sharp peak emerges near $r^{*} \approx r_{c}$ - we call this an "attraction-driven peak" as it is within the attractive range of the potential. The height of the peak is correlated with the strength of attraction $(\epsilon)$. However, the average distances (vertical lines in Fig. 1(c)) appear independent of $\epsilon_{i j}$. This difference is also reflected in the cumulative distribution function as shown in SI. Together, these results imply that average distances between bead-pairs may not represent the complete picture of chromatin organisation; understanding the whole distribution is necessary.

Given that we have the optimal interaction strengths that satisfy the experimentally known contact probability constraints [29], we can answer the following important question: Are the measurable properties of a given bead-pair (e.g. $r_{5,30}$ ) solely determined by the interaction between those two particular beads $\left(\epsilon_{5,30}\right)$ or are they influenced by the interactions among other beadpairs as well? To answer this, we adopted the following strategy: we systematically switched off the attractive interaction among certain bead pairs and computed probability distributions and other polymer properties. We simulated polymers for the following four cases: (i) all interactions are considered - GM12878 (OFF), (ii) only those interactions above $1 k_{B} T$ are considered - we call it OFF:GT1 - all weak interactions $\left(<1 k_{B} T\right)$ are switched off here, (iii) only strong interactions above $2 k_{B} T$ are considered - OFF:GT2 - all weak and medium interactions $\left(<2 k_{B} T\right)$ are switched off (iv) all attractive interactions are switched off - the SAW polymer. These four cases can be cosidered as four different epigenetic states - states having different interaction strengths due to underlying epigenetic variations. Fig. $1(\mathrm{~d})$ shows $p\left(r_{5,30}\right)$ for all the four cases. When we switch off the weak interactions below $1 k_{B} T$ (OFF:GT1), compared to the OFF state, the height of the interaction-driven peak of the distribution decreases and overall the polymer swells resulting in the shift of the second peak (compare pink and red curves in Fig. 1(d)). This implies that weak interactions having strengths comparable to thermal fluctuations can also influence the contact probability and polymer configurations. If we keep only the highly prominent interactions and neglect all interactions below $2 k_{B} T$ (OFF:GT2), the interaction-driven peak further diminishes and the distribution function approaches the SAW distribution (compare blue with other curves in Fig. 1(d)). Note that the interaction between beads 5 and 30 is present $\left(\epsilon_{5,30}=2.09\right)$ in all the cases except in the SAW case. These results suggest that the measurable properties for a given bead-pair (e.g. $r_{5,30}$ ) depends not only on the attraction strength of that particular bead pair but also on the interactions of the whole polymer chain. This result implies that all bead-pairs collectively/cooperativily contribute in determining the relative position for a particular bead-pair.

\section{Epigenetic changes alter the volume and shape of the chromatin domains}

The above picture suggests that the chromatin folding is influenced by collective behavior of all beads having different interaction strengths. To examine the nature of collective behavior, we probed a property of the whole polymer namely the radius of gyration $\left(R_{g}\right)$ defined in SI. To understand how folding is affected by different epigenetic states, we did the following. We started with a polymer having no interactions (SAW), added weak interactions (small $\epsilon$ ) that exist between beads in the OFF state as the first step, equilibrated, computed $R_{g}$ and sequentially added stronger interactions between beads step by step $(\epsilon<0.5, \epsilon<1.0, \ldots, \epsilon<2$ and so on, denoted as LT1, LT2 etc), until the OFF state (GM12878) is reached. Each step was equilibrated and $R_{g}$ was computed (see Fig:2, top panel). From $R_{g}$, we have also computed the volume $V=(4 / 3) \pi R_{g}^{3}$ of the chromatin domain as shown in the right side $y$-axis. As seen from the figure, adding very weak interactions does not change the $R_{g}$ much. However, adding intermediate interactions significantly reduces the $R_{g}$ and it saturates as the interactions gets stronger, resulting in a sigmoidal-like curve showing signatures of cooperative/collective behavior. Since, we have equilibrated the polymer for each set of $\epsilon$ values, the LHS of the curve can be interpreted in two ways: folding the polymer by adding stronger and stronger interaction starting with a completely unfolded state or equivalently unfolding the polymer by removing 
bioRxiv preprint doi: https://doi.org/10.1101/2021.02.17.431616; this version posted May 11, 2021. The copyright holder for this preprint (which was not certified by peer review) is the author/funder, who has granted bioRxiv a license to display the preprint in perpetuity. It is made available under aCC-BY-NC-ND 4.0 International license.

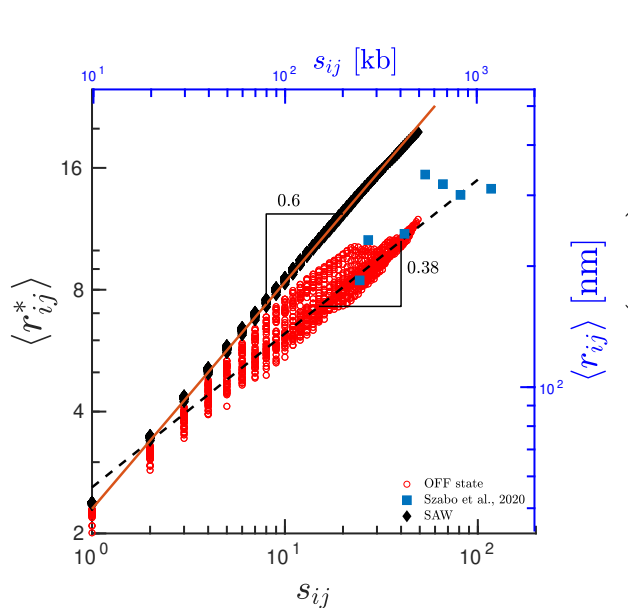

(a)

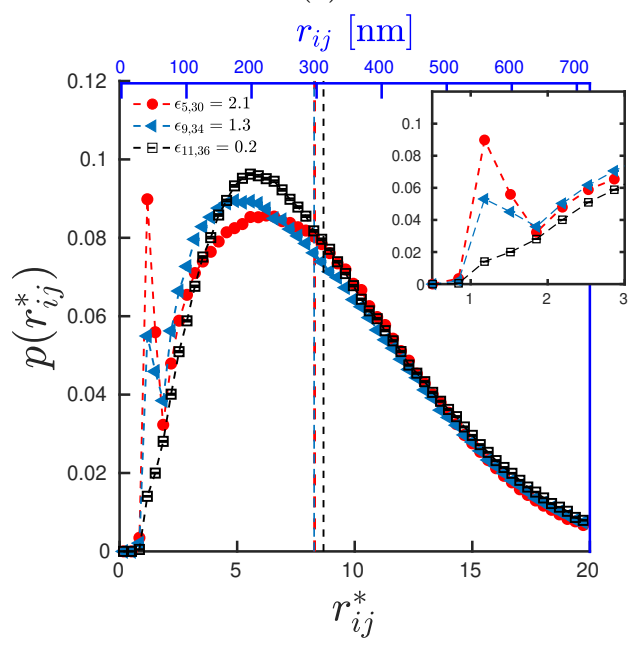

(c)

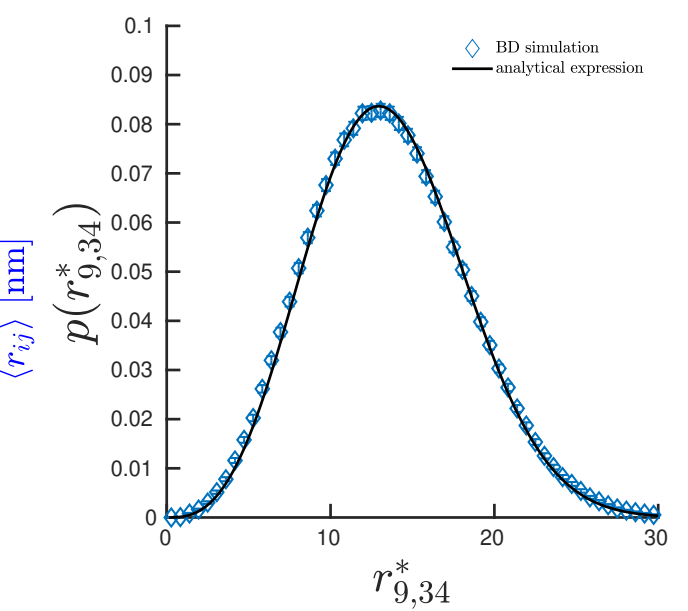

(b)

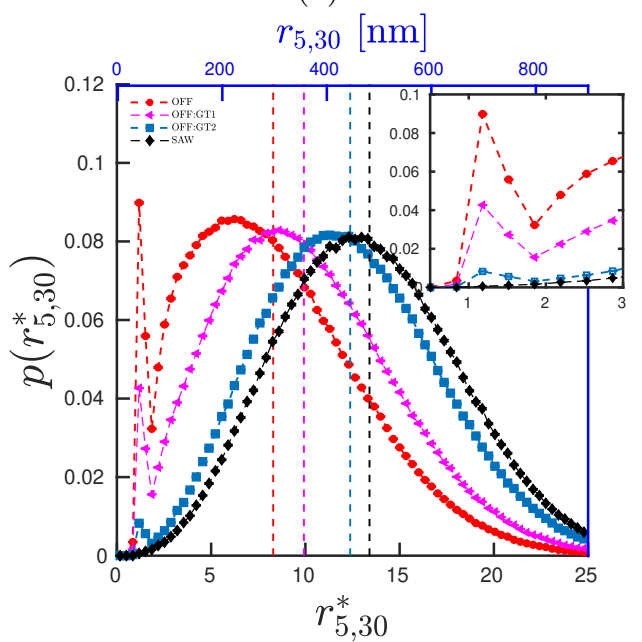

(d)

FIG. 1: Changes in distance probability distribution $\left(p\left(r_{i j}^{*}\right)\right)$, cooperative nature of chromatin folding: (a) Average 3D distances between all bead-pairs as a function of corresponding genomic distances for the control simulations (SAW, black symbols), chromatin domain that we simulated (red symbols) and comparison with experimental data from [12] (blue symbols). The major axes (lower $x$ and left $y$ ) represent quantities in dimensionless units (see methods) while the other axes (upper $x$ and right $y$ ) represent the same in standard units. (b) Distance probability distribution $p\left(r_{i j}^{*}\right)$ from simulations compared with the analytical expression [46] for a pair of beads 9 and 34 of a SAW polymer of total length $N=50$. (c) $p\left(r_{i j}^{*}\right)$ for various bead pairs with different interaction strengths $\epsilon_{i j}$, but same $s_{i j}=25$ in the OFF state of $\alpha$-globin gene. The interaction-driven peak is highlighted in the inset. Vertical dashed lines represent $\left\langle r_{i j}^{*}\right\rangle$ corresponding to each distribution. (d) Comparison of $p\left(r_{i j}^{*}\right)$ between different "epigenetic states". OFF: state with all interactions in GM12878 (red), OFF GT1: when weak interactions are ignored; i.e., only with $\epsilon_{i j}>1 k_{\mathrm{B}} T$ interactions (pink), OFF GT2: when only very high interactions $\left(\epsilon_{i j}>2 k_{\mathrm{B}} T\right)$ are accounted for (blue), SAW: control simulation with no attractive interaction (black). Vertical lines and inset are similar to (c).

the stronger interaction starting with a completely folded OFF state. One can also ask how the polymer would fold if one adds strong interactions (larger $\epsilon$ ) as the first step, starting with SAW, and then add weaker interaction sequentially step by step (denoted as GT1, GT2 etc). This is shown in the RHS of Fig. 2 (blue symbols). The whole curve suggests that having prominent interactions alone or weaker interactions alone may not take the system closer to its full equilibrium state. We also show typical snapshots of $3 \mathrm{D}$ chromatin configurations corresponding to different epigenetic states. As expected, the OFF state is compact and the volume of the domain increases as we go towards the SAW state. The approximate two foldchange in volume between the two extreme states seen here are roughly the same order as the density change observed experimentally [47].

To quantify how the shape of the chromatin domain changes with epigenetic states, we computed the asphericity $(\mathrm{B})$ and the acylindricity $(\mathrm{C})$ parameters (see SI for definition). Asphericity quantifies the extent of devi- 


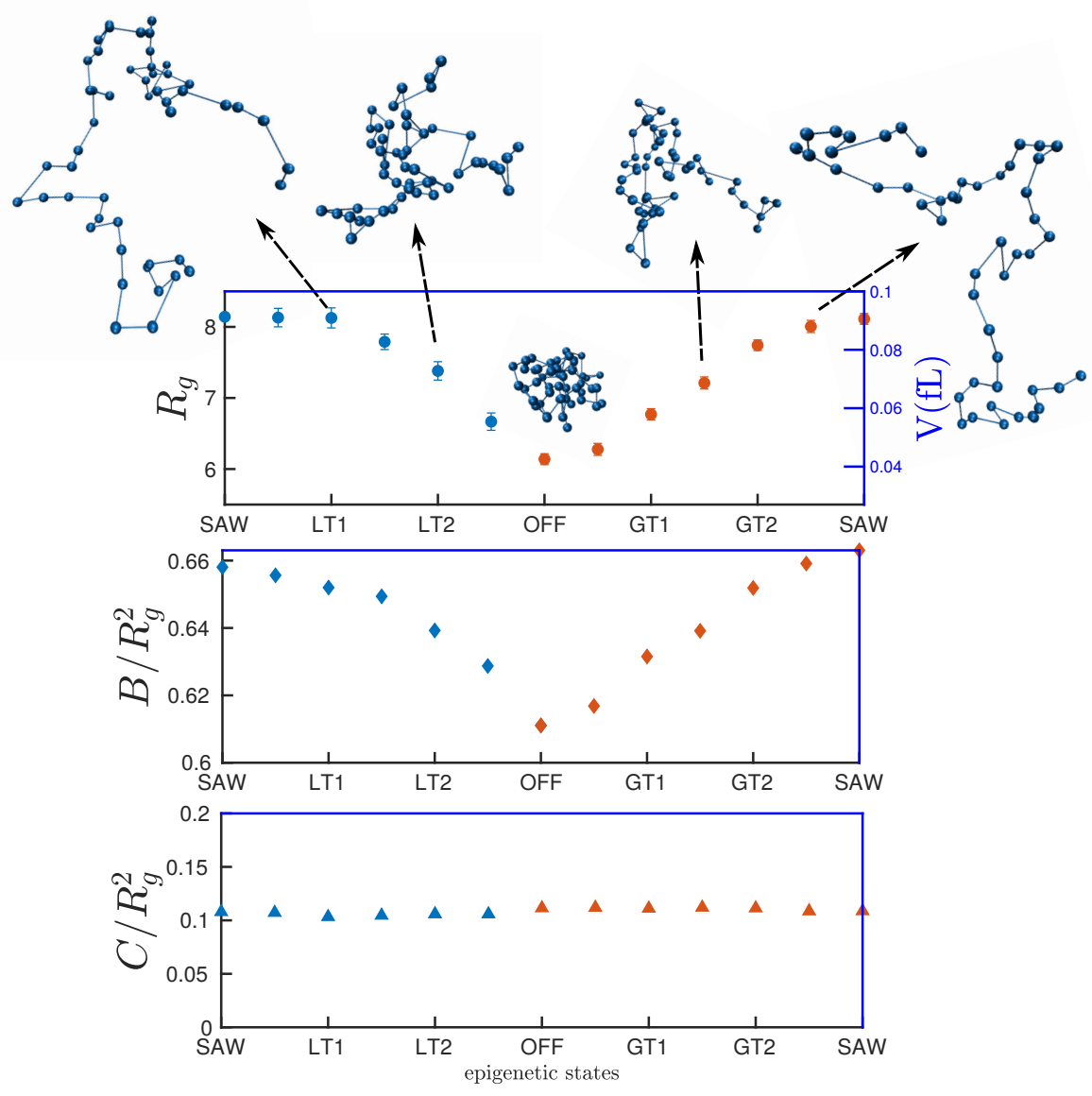

FIG. 2: Interaction strengths determining shape properties of chromatin domain: Upper panel: Size of the chromatin domain $\left(R_{g}\right)$ as we perturb interaction strengths. $x$-axis represents different interaction states with extreme ends representing the control (SAW) polymer and the OFF state in the middle. LT1 (LT $x$ ) indicates that all interactions below $1 k_{B} T\left(x k_{B} T\right.$ ) are present in the polymer. Similarly GT1 (GT $x)$ indicates that all interactions above $1 k_{B} T\left(x k_{B} T\right)$ are present. The right $y$-axis indicates volume of the chromatin domain in femtolitre. Snapshots from simulations at various epigenetic states are shown around the perimeter of the graph. Bottom two panels represent the normalized asphericity $\left(B / R_{g}^{2}\right)$ and acylindricity $\left(C / R_{g}^{2}\right)$, respectively. See SI for the definition of $B$ and $C$. The $x$-axis is the same in all panels.

ation from a spherical shape. If a polymer is coiled with the average shape of a sphere, $B=0$. Here a positive $\mathrm{B}$ value suggests that even in the OFF state, the chromatin domain is not a perfect sphere. As we go from OFF to SAW, the asphericity increases by $\approx 65 \%$ as shown in SI. However, the asphericity scaled with the polymer size $\left(B / R_{g}^{2}\right)$ changes by $\approx 10 \%$. Similar to $R_{g}$, we have shown the GT (RHS, orange symbols) and LT (LHS, blue symbols) cases for the asphericity too. Even though both sides are monotonically increasing, note that LT cases are not equivalent to the GT cases. We also compute the acylindricity parameter that quantifies the extent of the deviation from a perfect cylinder. Here too, $C>0$ values suggest that the chromatin domain is not a perfect cylinder (see the lower panel of Fig. 2 and SI). Even though the acylindricity is monotonically increasing (shown in SI) from the OFF state to a SAW, it is increasing in proportion to the size of the polymer. Hence the scaled acylindricity $\left(C / R_{g}^{2}\right)$ is nearly a constant as shown in the lower panel of Fig. 2.

\section{Estimation of stiffness and drag properties from domain relaxation times and fluctuations}

Whether chromatin is liquid-like, solid-like or gel-like has been a matter of intense discussion in the recent literature $[10,24,48,49]$. In the phase separation picture, chromatin segments are thought to be "liquid-like", dynamically exploring various configurations. Given that our model can study the stochastic nature of formation and breakage of bonds, and polymer dynamics, consistent with what is observed in $\mathrm{Hi}-\mathrm{C}$ experiments, below we compute relaxation times and fluctuations of the chromatin domain and estimate effective elastic and drag properties. 
bioRxiv preprint doi: https://doi.org/10.1101/2021.02.17.431616; this version posted May 11, 2021. The copyright holder for this preprint (which was not certified by peer review) is the author/funder, who has granted bioRxiv a license to display the preprint in perpetuity. It is made available under aCC-BY-NC-ND 4.0 International license.

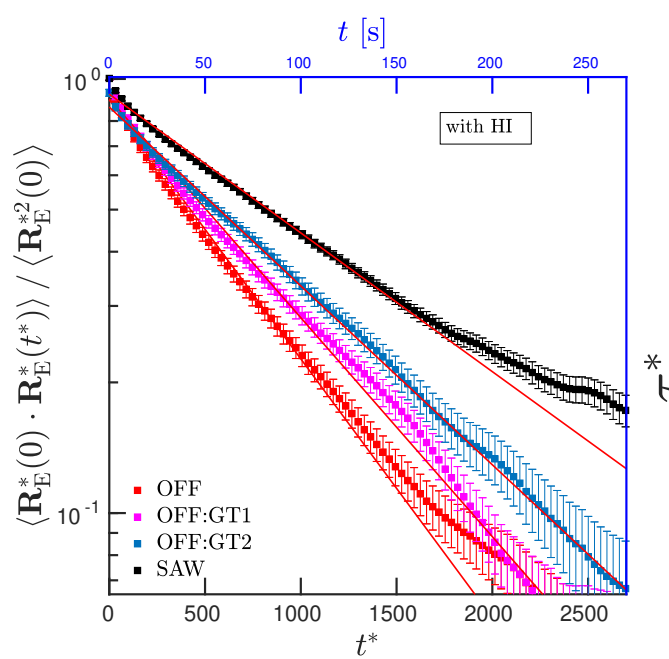

(a)

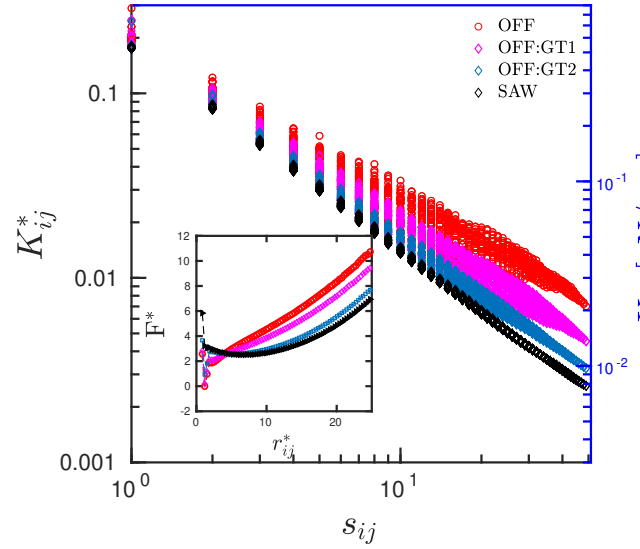

(c)

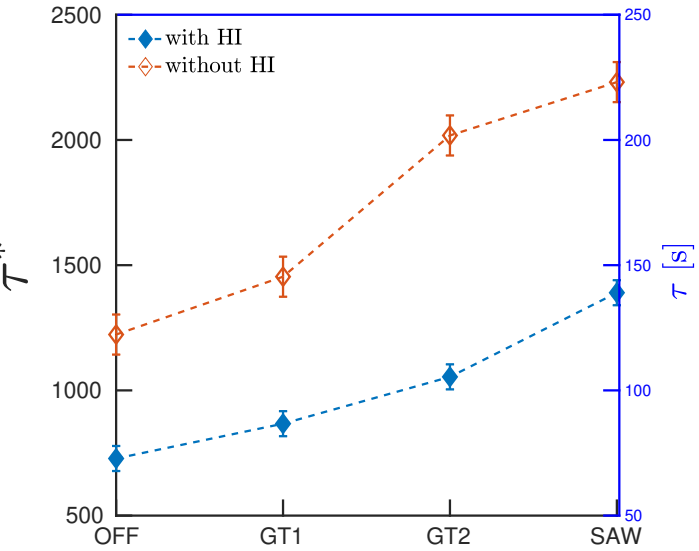

(b)

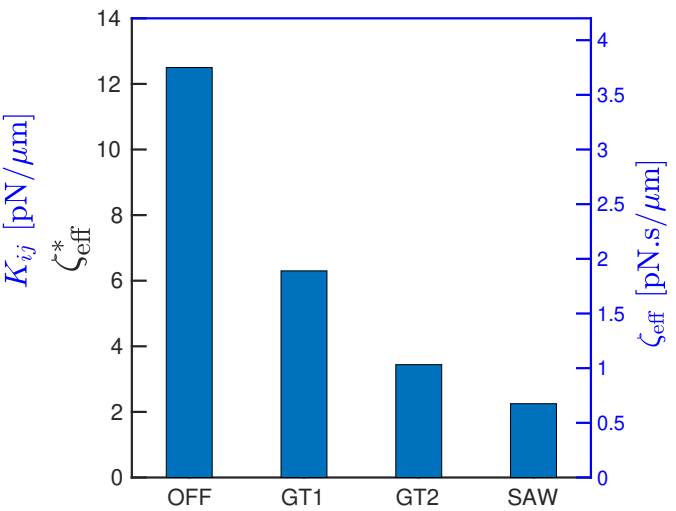

(d)

FIG. 3: Stiffness and relaxation time characterising domain properties: (a) Exponential decay of end-to-end autocorrelation function with time for four epigenetic states computed with HI. (b) Relaxation times $(\tau)$ with and without HI reveal that HI helps in relaxing the polymer faster. (c) Effective stiffness between all the bead-pairs; OFF state is more stiff compared to less interacting states and SAW. Inset: Free energy as a function of bead pair distance $r_{5,30}$. (d) Effective viscous drag felt by different chromatin states. OFF state chromatin (with stronger interactions) is more stiff and has a higher viscous drag.

First, we computed the end-to-end vector autocorrelation function $\left\langle\mathbf{R}_{\mathrm{E}}^{*}(0) \cdot \mathbf{R}_{\mathrm{E}}^{*}\left(t^{*}\right)\right\rangle /\left\langle\mathbf{R}_{\mathrm{E}}^{* 2}(0)\right\rangle$ where $\mathbf{R}_{\mathrm{E}}^{*}=$ $\left(\mathbf{r}_{1}^{*}-\mathbf{r}_{50}^{*}\right)$ and extracted the longest relaxation time $\tau^{*}$ with and without HI. The autocorrelation decay computed with HI is shown in Fig. 3(a) and no-HI case is shown in SI. Fig. 3(b) shows that the relaxation times for all the epigenetic states are lower with HI, as observed previously for the protein folding simulations [50, 51]. We also computed $R_{g}$ autocorrelation function and observed the similar behaviour. All results presented in this paper are computed with HI, unless stated otherwise. The chromatin in the OFF state has a lower relaxation time compared to a SAW chromatin (no attractive interaction). Even though in a different context, a similar behavior has also been observed in recent experiments $[10,52]$. To understand this behavior, we investigate the elastic and drag properties of the chromatin domain. From the measurement of fluctuations of each bead-pair we can compute an effective stiffness defined as $K_{i j}=k_{\mathrm{B}} T /\left\langle\left|\mathbf{r}_{i}-\mathbf{r}_{j}\right|^{\mathbf{2}}\right\rangle$ (Fig. 3(c)). As expected, the highly interacting OFF state is more stiff than the other epigenetic states including SAW. This can also be understood from the free energy as a function of bead pair distance $F^{*}\left(r^{*}\right)=-\ln \left(p\left(r^{*}\right) / 4 \pi r^{* 2}\right)$ (see inset). The above behaviour is consistent with $K_{i j}^{*} \sim \frac{\partial^{2} F_{i j}^{*}}{\partial r_{i j}^{2}}$ and stiffness $\left(K_{i j}^{*}\right)$ of different epigenetic states do show similar behaviour. Since timescales in such problems are inversely proportional to the stiffness, the observed lesser time is explained by the higher stiffness. For the known stiffness and relaxation times, we can compute an effective drag coefficient defined as $\zeta_{\text {eff }}^{*}=\tau^{*} \times K^{*}$. Taking the effective stiffness of the end beads $\left(K_{1,50}^{*}\right)$, we find that the drag for the OFF state is higher than the other states 
bioRxiv preprint doi: https://doi.org/10.1101/2021.02.17.431616; this version posted May 11, 2021. The copyright holder for this preprint (which was not certified by peer review) is the author/funder, who has granted bioRxiv a license to display the preprint in perpetuity. It is made available under aCC-BY-NC-ND 4.0 International license.

suggesting that the existence of larger attractive interactions reduces its ability to reorganize. Both the stiffness and drag are greater for the OFF state than the SAW, but they combine to lead to a faster relaxation time for the OFF state. Our findings are consistenent with the recent experimental report that highly interacting chromatin shows reduced mobility as measured by Fluorescence Recovery After Photobleaching (FRAP) technique, revealing the gel-like nature of chromatin [24, 49].

\section{Interplay between interaction energy and polymer entropy influences the dynamics of chromatin domain}

While we have gained insights into steady state fluctuations and distance distributions, how the interactions would affect chromatin dynamics can be further probed [53, 54]. We know that contacts between chromatin segments are dynamic; proteins that form contacts bind and dissociate resulting in stochastic formation and breakage of contacts. This opens up interesting questions: How long do two beads remain in contact (looped)? When loops break and beads diffuse away, how long does it take for the bead pairs to come back in contact? What are the factors (interaction strengths, polymer entropy etc.) dictating the phenomena of dynamic contacts?

To study the temporal nature of chromatin, we define loop formation time $\left(t_{\mathrm{L}}^{*}\right)$ and contact time $\left(t_{\mathrm{C}}^{*}\right)$ for all bead-pairs. $t_{\mathrm{L}}^{*}$ is defined as the time taken for a pair of beads to meet $\left(r_{i j}^{*}<r_{\mathrm{C}}^{*}\right)$ for the first time, starting from a random equilibrium configuration. $t_{\mathrm{C}}^{*}$ is defined as the duration that the bead-pairs remain looped/in contact. A schematic representation of a typical time trajectory of $3 \mathrm{D}$ distance indicating $t_{\mathrm{L}}^{*}$ and $t_{\mathrm{C}}^{*}$ is shown in Fig. 4(a) and the actual data from our simulation, as an example, is shown in SI. Corresponding average quantities are defined by $\left\langle t_{\mathrm{L}}^{*}\right\rangle$ and $\left\langle t_{\mathrm{C}}^{*}\right\rangle$, respectively.

Two possible factors that can influence these temporal quantities are interaction strengths $(\epsilon)$ and polymer entropy. Since two beads having a larger segment length between them will have a higher entropy, it is expected that the time to come into contact is longer. In other words, the time of looping is expected to be dictated by polymer entropy. To validate this hypothesis, we looked at $\left\langle t_{\mathrm{L}}^{*}\right\rangle$ as a function of the genomic length with and without HI.

As shown in Fig. 4(b) $\left\langle t_{\mathrm{L}}^{*}\right\rangle$ monotonically increases with $s$ showing a power law behavior $\left\langle t_{\mathrm{L}}^{*}\right\rangle \sim s^{\mu}$. As a control, we matched our $\left\langle t_{\mathrm{L}}^{*}\right\rangle$ results with the previously known exponents $\mu \approx 2.3$ for SAW and $\mu=2.0$ for a random polymer (see top inset) [55]. By simulating various chain lengths $(N=10,20, \ldots)$ we can infer that the deviation from the power law for large $s$ is due to finite chain effects (top inset). We have also computed $\left\langle t_{\mathrm{L}}^{*}\right\rangle$ for all the other epigenetic states revealing $2.3<\mu \leq 1.4$.
The OFF state having all interactions shows the smallest exponent of 1.4. As we remove interactions from the system, $\mu$ gradually approaches the SAW limit. The scaling appears independent of $\mathrm{HI}$ as shown in SI. The change in power law may also be understood by looking at the free energy plotted in Fig. 3(c) inset. One can see that the free energy has a higher tilt in the OFF state compared to the other states, implying that the bead-pairs can move along the landscape quicker in the OFF state. The results for $\left\langle t_{\mathrm{L}}^{*}\right\rangle$ suggests that even in the absence of loop extrusion, the looping time is not too long (seconds to minutes). This also indicates that the micro phaseseparation could be a viable mechanism for bringing together chromatin segments and possibly explains the experimentally observed fact that chromatin is functional even in the absence of loop extruding factors [11, 20, 23]. We then examined how the interaction strength influences $\left\langle t_{\mathrm{L}}^{*}\right\rangle$, and found that there is a huge spread in the $\left\langle t_{\mathrm{L}}^{*}\right\rangle$ values, for a given $\epsilon$ (Fig. $4(\mathrm{c})$ ), with the average showing a mild dependence on $\epsilon$ (inset).

Interestingly the values of $\left\langle t_{\mathrm{C}}^{*}\right\rangle$ are nearly independent of genomic separation (Fig. 4(d)). Here too there is a huge variability among different bead pairs with the inset showing the behaviour when the segment length is averaged over all pairs having the same $s$. However, the interaction strength significantly alters the $\left\langle t_{\mathrm{C}}^{*}\right\rangle$ (Fig. 4(e)) showing an exponential increase. This suggests that the $\left\langle t_{\mathrm{L}}^{*}\right\rangle$ is determined by the interplay between entropy (resulting from genomic separation) and energy (interaction strength). Once bead-pairs come in contact, $\left\langle t_{\mathrm{C}}^{*}\right\rangle$ is dominated by the interaction strength.

For the OFF and SAW states, we also show $\left\langle t_{\mathrm{L}}^{*}\right\rangle$ between all pairs of beads as a heatmap (see Fig. 4(f) \& (g)). One can quickly note that the range of SAW time scales is much higher than that of the OFF state. This is the consequence of higher $\mu$ for the SAW compared to the OFF state. In the SAW, one can observe that the times are similar for all points having the same distance away from the diagonal (a line parallel to diagonal axis), suggesting that what matters in this case is the inter bead distance $(s)$. In contrast, in the OFF state, there is a heterogeneity and curvy color contours suggesting that the time values are not just a function of segment length alone but also the identity (interaction strength) of the individual bead pairs. This once again points to the interplay between entropy and energy.

\section{Nature of loop formation and contact time distributions}

So far we have studied the average loop formation times and contact times; however, should one assume that the average values describe these quantities completely? To answer this, similar to $p\left(r^{*}\right)$, here we have investigated the nature of the distribution of the tem- 


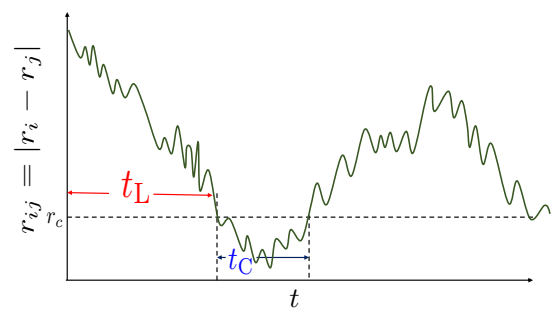

(a)

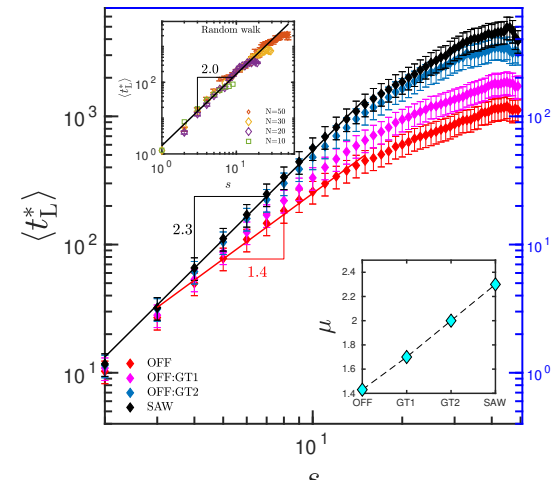

(b)

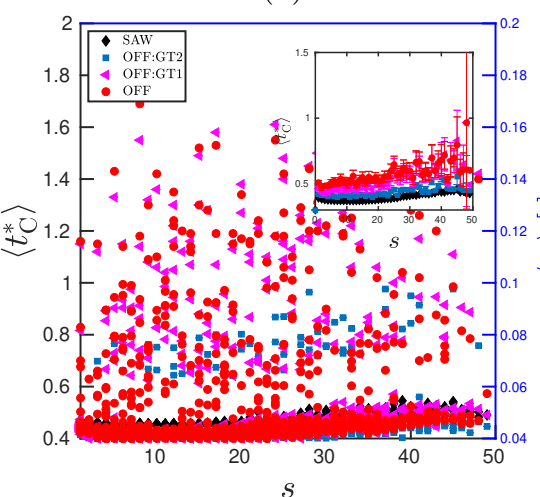

(d)

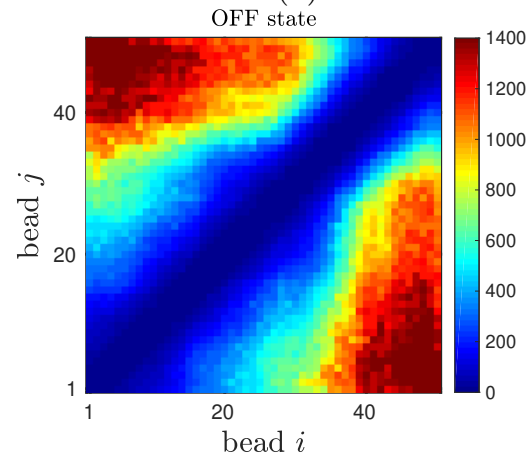

(f)

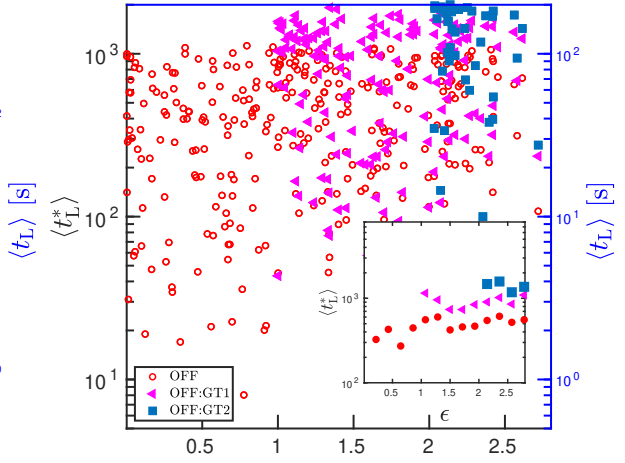

(c)

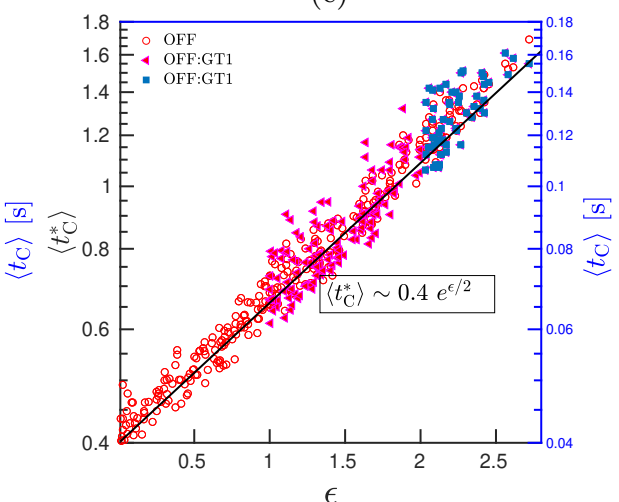

(e)

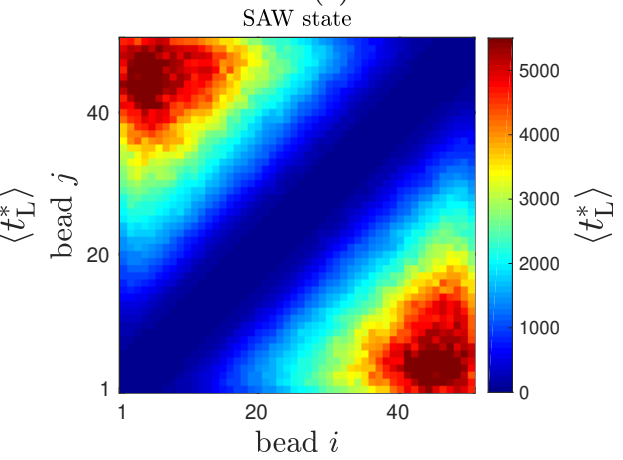

$(\mathrm{g})$

FIG. 4: Interaction energy and genomic seperation dictate the behavior of loop formation time $\left\langle t_{\mathrm{L}}^{*}\right\rangle$ and contact time $\left\langle t_{\mathrm{C}}^{*}\right\rangle$ : (a) schematic representation of the distance between two beads in a single trajectory showing $t_{\mathrm{L}}^{*}$ and $t_{\mathrm{c}}^{*}$. (b) $\left\langle t_{\mathrm{L}}^{*}\right\rangle$ has a power law scaling with genomic length $\left(\left\langle t_{\mathrm{L}}^{*}\right\rangle \sim s^{\mu}\right.$ ) with exponent varying from 1.4 (OFF state) to 2.3 (SAW) for different chromatin states. The exponents are shown in lower inset. The upper inset shows $\left\langle t_{\mathrm{L}}^{*}\right\rangle$ for various $N$ values for a random walk polymer. (c) $\left\langle t_{\mathrm{L}}^{*}\right\rangle$ as a function of interaction strength with each point representing a bead pair. Note the huge spread in $\left\langle t_{\mathrm{L}}^{*}\right\rangle$. Inset: $\left\langle t_{\mathrm{L}}^{*}\right\rangle$ binned and averaged over all bead pairs having same $\epsilon$ showing minimal influence of $\epsilon$. (d) $\left\langle t_{\mathrm{C}}^{*}\right\rangle$ as a function of $s$ with each point representing a bead pair. Here too, note the spread. Inset: $\left\langle t_{\mathrm{C}}^{*}\right\rangle$ binned and averaged over all bead pairs having the same $s$ showing minimal dependence on the segment length. (e) $\left\langle t_{\mathrm{C}}^{*}\right\rangle$ increases exponentially with the interaction strength. $\left\langle t_{\mathrm{L}}^{*}\right\rangle$ for all the bead pairs as a heatmap for (f) OFF and (g) SAW states.

poral quantities. In Fig. 5(a) and (b), we present the probability distributions of contact $\left(p\left(t_{\mathrm{C}}^{*}\right)\right)$ and loop formation $\left(p\left(t_{\mathrm{L}}^{*}\right)\right)$ times, respectively. We observe that $p\left(t_{\mathrm{C}}^{*}\right) \sim \exp \left(-t_{\mathrm{C}}^{*} / \tau_{c}\right)$ with the average time $\tau_{c}$ that depends on the epigenetic state (SAW: $\tau_{c}=1 / 1.6$, OFF: $\left.\tau_{c}=1 / 1.25\right) . \quad \tau_{c}$ is small for the SAW and it increases as we add interactions to the system. However, interest- ingly, the probability of loop formation time $\left(t_{\mathrm{L}}^{*}\right)$ has a power law decay $\left(p\left(t_{\mathrm{L}}^{*}\right) \sim\left(t_{\mathrm{L}}^{*}\right)^{-\gamma}\right)$. This suggests that there is a huge diversity in loop formation times, and the average looping time alone may not be sufficient to describe the loop formation phonomena. We find that the epigenetic states alter the slope of the distribution (SAW: $\gamma=0.4$, OFF: $\gamma=1.0)$ keeping the overall nature the 
bioRxiv preprint doi: https://doi.org/10.1101/2021.02.17.431616; this version posted May 11, 2021. The copyright holder for this preprint

same. Comparison of these two distributions reveals that quantitatively the $t_{\mathrm{L}}^{*}$ is much larger than $\tau_{c}^{*}$, indicating that chromatin segments take longer to come into contact but stay in contact for a short time.

\section{Suggestions for experiments to test our predictions}

Since we take HiC-like data as input and predict positional fluctuations and dynamics of chromatin segments, microscopy is the ideal method to test our predictions [10, 11, 52]. All $p(r)$ predictions (Fig. 1) may be tested either via live (without fixing) microscopy experiments or by collecting a large number of frozen snapshots of segment-locations via FISH or equivalent methods. Imaging experiments may also estimate the volume occupied by a domain (Fig. 2). From the positional fluctuation data, one can also obtain the effective stiffness as described earlier in this paper (Fig. 3). To measure the time-dependent quantities (Fig. 4, Fig. 5), apart from live microscopy experiments, one may also design appropriate FRET pairs that can probe quantities like the contact time [56]. Obtaining all of these quantities for different epigenetic states would facilitate comparison with our predictions.

\section{DISCUSSION}

In this paper, we have investigated the dynamics of chromatin domains, using an inverse Brownian dynamics model including hydrodynamic interactions, and presented the corresponding results. In this section, we discuss several aspects of the model and results in greater detail. In particular, we would like to highlight the novel aspects of our model in the context of earlier work in this area. Most of the existing models have been addressing the forward problem of obtaining 3D configurations of chromatin given a certain set of parameters including interaction strengths $[19,40,57,58]$. While some of the models study nucleosome assembly at the scale of a few nucleosomes (a few $\mathrm{kb}$ ), other models investigate $3 \mathrm{D}$ organization on the lengthscale of a domain (of the order $\mathrm{Mb}$ ) or a whole chromosome. Spakowitz and coworkers studied spreading of histone modifications and how histone marks could drive formation of heterochromatin $[40,57]$. Mirny and coworkers investigated different polymer aspects of chromatin folding; they showed that certain combinations of intra-chromatin and interchromatin interactions could lead to very different chromatin organisations such as that of inverted nuclei [19]. While most of the above models focused on statics of chromatin, there have been a few studies investigating the dynamics. Dudko and coworkers studied various first passage processes in the genome, in the B-lymphocytes in particular. While they show that a fractional Langevin equation describing two beads in a sphere could explain some of the experimentally observed features, they also investigate the dynamics using forward simulation of a polymer model $[54,59]$.

In spite of all these studies, there have been only a few iterative/inverse models that obtain optimal parameters based on available experimental data such as HiC or Chip-Seq. Di Pierro et al. [33] have presented a model where the $3 \mathrm{D}$ organization of the chromatin is predicted by obtaining the interaction parameters using a maximum entropy method. Here the authors do not use the Hi-C data to compute interaction strengths but rather the epigenetic (histone modification/protein binding) data. Thirumalai and coworkers have studied a polymer model and extracted mean 3D distances and standard deviations between all bead pairs from $\mathrm{Hi}-\mathrm{C}$ matrix [60]. Then they predict an ensemble of 3D configurations consistent with the above parameters. It differs from our approach since it optimizes spatial distances and not the interaction strengths. Similarly, there have been other models that convert $\mathrm{Hi}-\mathrm{C}$ contact map to the corresponding spatial distance by assuming an inverse relation [61]. Nicodemi and coworkers have presented a coarse-grained polymer model explicitly accounting for binder proteins; they obtain the optimal number of protein types and binding sites that matched with $\mathrm{Hi}-\mathrm{C}$ data [37]. While this model has the details about the protein binding locations, it does not optimise for the interaction strengths directly; once they obtain the optimal number of protein types and bindings sites, the concentration and interaction strengths of proteins are varied as separate parameters. All the above methods make an impressive attempt to address common issues and have used different approaches. Here, we not only use an inverse method to derive optimal interaction strengths, but also compute dynamics, incorporate HI, and make novel predictions such as double-peaked distance distributions and relaxation times of different epigenetic states.

Since many methods use epigenetic datasets to model chromatin, we would like to argue that using Hi-C data alone has the following advantage. In many a context for example when studies are done under different drug treated conditions - we may not have the epigenetic data. We may not know the precise protein concentrations, affinities of proteins, organization of proteins and histone marks under all these conditions. Hence obtaining optimal intra-chromatin interaction strengths for all bead pairs with Hi-C data alone has its advantage and may be useful to study various cell states. In this work given the contact probabiity data - without any further information - we predict the intra-chromatin interactions that are a net result of different epigenetic marks, their intensities, different protein/DNA interactions and distributions/concentrations of proteins.

Another point worthy of discussion is whether considering a small domain is justifiable or not. First of all, 


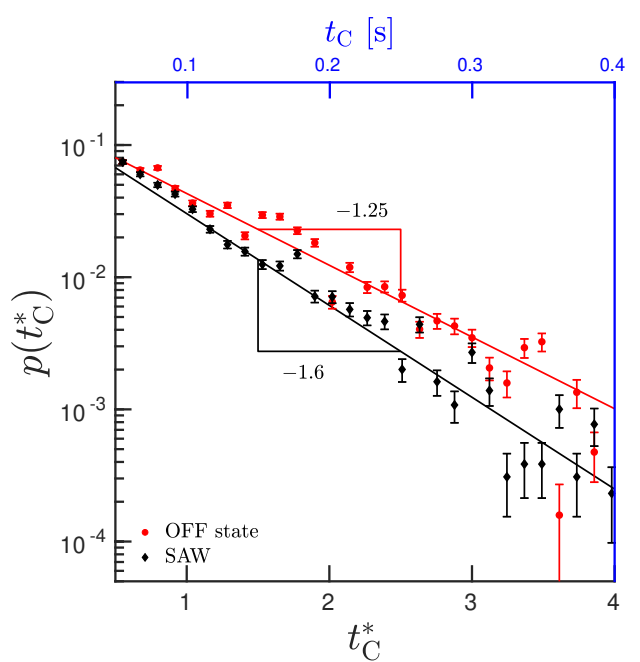

(a)

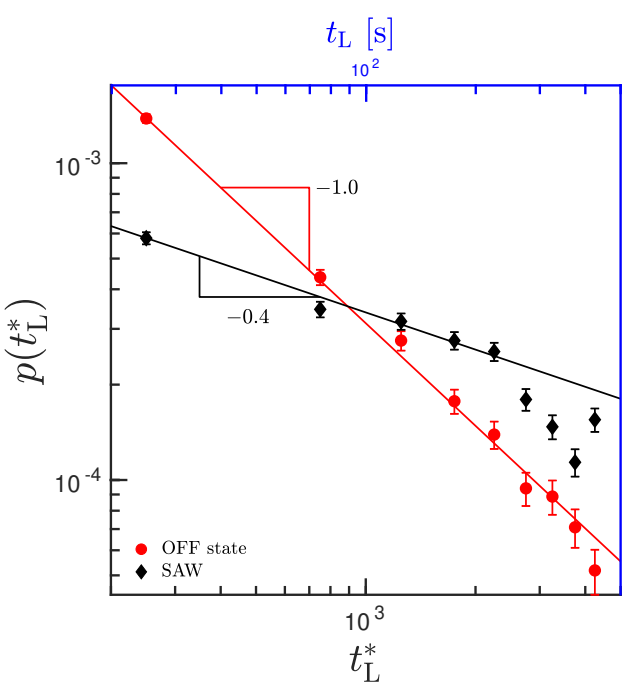

(b)

FIG. 5: Distribution function for contact time $\left(t_{\mathrm{C}}^{*}\right)$ and loop formation time $\left(t_{\mathrm{L}}^{*}\right)$ for a specific bead-pair (bead 5 and bead 30$)$ in SAW and OFF state are shown in (a) and (b), respectively.

it is computationally expensive to simulate the whole chromatin at higher resolution with hydrodynamic interactions; moreover, from a biology point of view there are sound arguments that suggest that simulating a small domain is reasonable. From the chromatin contact probability data, we know that chromatin is organised into multiple domains (also often called TADs). This data suggest that one domain has much less probability (negligible probability) to interact with another domain. Hence their inter-domain interaction $(\epsilon)$ can be assumed to be negligible. Since our aim here is to probe the dynamics of beads within a domain, it is reasonable to simulate a single domain alone. Also note that the gene regulation and cellular processes are thought to be happening within a domain. Interestingly, recent experimental data has shown that when the chromatin polymer is cut and each domain is separated out, most of those domains are stable and behave very similar to how they behaved within the whole chromatin indicating that each domain is independent [15]. This justifies our investigation of each chromatin domain as separate entity.

One of the important conclusions of polymer physics is that the temporal quantities are greatly affected by HI $[50,51,62-69]$. The influence of the effect of HI on the chromatin domain is a question which no one has attempted until now. To the best of our knowledge, this work is the first attempt to study the chromatin dynamics with HI. Here, we show that while the relaxation times do depend on HI, other quantities on the length scale of the domain remain less affected by HI. However, there are interesting questions that remain to be addressed on how HI affects dynamics in a crowded environment, given that the concentration of macromolecules in a cell is in an unentangled semi-dilute regime, where HI is known to be important. However, investigating dynamics in a crowded environment is a difficult problem given that $\mathrm{HI}$ is a long-ranged and many-body interaction. The problem of the role of screening of $\mathrm{HI}$ is also important in this context. Our current study could be a first step towards this direction.

Quantities calculated here have immense physical and biological significance. The finding of two peaks in the distribution function is a novel aspects that can have ramifications. As mentioned earlier, there is an ongoing debate in the field about whether the gene regulation requires actual physical contact between two regulatory segments or only the proximity would suffice. Cellular processes such as transport of proteins from one region to another (eg. enhancer-promoter), spreading of histone modifications in the 3D space etc would crucially depend on $p(r)$. For example, given $r$, one can compute the time $\left(\tau_{p}\right)$ for proteins/enzymes to diffuse from location $r_{i}$ to $r_{j}$. The mean time would depend on the distribution as $\left\langle\tau_{p}\right\rangle=\int \tau_{p} p(r) d r$. However, apart from the distance among segments, the accessibility would depend on the local compactness and diffusivity too. That is, compactness of the domain (Fig. 2) and effective viscous drag (Fig. 3) together with $p(r)$ (Fig. 1) would be crucial for understanding how physics of chromatin would affect biological function. Given that phase separation is argued to be one of the important factors determining domain formation, our study reveals how the interplay between epigenetic states and polymer dynamics would affect loop formation and contact times. 
bioRxiv preprint doi: https://doi.org/10.1101/2021.02.17.431616; this version posted May 11, 2021. The copyright holder for this preprint

\section{CONCLUSION}

Even though there is a great improvement in our understanding of static nature of chromatin organization, very little is known about the dynamics, which is a crucial aspect of in vivo chromatin. With the advancement of technology, it is now possible to experimentally probe fluctuations and dynamics of chromatin polymer. However, the main challenge to simulate dynamics of chromatin is that we do not know the interaction strength parameters among different segments. We have overcome this challenge by using an inverse technique and obtained optimal interaction strengths between all chromatin segments and used it to investigate the dynamics of a chromatin domain.

We summarize our key findings: (i) The nature of 3D spatial distances between chromatin segments in a domain, obtained from simulations, are comparable to recent experimental findings. (ii) Going beyond the average properties, we computed the distance probability distribution and it shows two peaks - an interaction-driven peak and an entropy-dominated peak. (iii) Assuming that interactions are arising from epigenetic states, we show how perturbations in epigenetic states would alter $p(r)$; the distance distribution between a given bead pair depends on the interaction strength of all other pairs suggesting the cooperative nature of chromatin folding. (iv) Volume and the shape properties of the chromatin domain depends on the epigenetic state. The OFF state is highly collapsed/compact, more spherical compared to the extended, less spherical SAW. (v) The relaxation time of the domain is dependent on the epigenetic state of the domain. Counter-intuitively, the relaxation time of a highly interacting OFF state is much smaller than that of a non interacting SAW polymer. We explain this phenomenon by computing effective stiffness of the domain from polymer fluctuations. We also show that the OFF state has a higher effective drag. (vi) We study dynamics accounting for crucial hydrodynamic interactions; we show that HI has a significant influence on the relaxation time of the chromatin domain. With HI, the domain takes half the time to relax as compared to the no-HI case. (vii) We compute the loop formation time and the time for the looped bead pairs to remain in contact. We show that average looping time has different scaling with genomic separation, depending on the epigenetic nature of the chromatin states. The looping times show a power law distribution indicating multiple timescales that might be involved with looping. On the other hand, the contact time has an exponential distribution.

This study can be further extended genomewide to examine various gene loci and investigate fluctuations and dynamics of all domains in the genome. Such polymer models are useful for examining aspects like spread of histone modifications and accessibility of the domains. We hope that this study would catalyse new experimental and computational studies examining the interplay between epigenetics and polymer dynamics.

\section{ACKNOWLEDGMENTS}

We thank Burkhard Dünweg, Dibyendu Das and Rajarshi Chakraborty for enlightening discussions. The work was supported by the MonARCH and SpaceTime computational facilities of Monash University and IIT Bombay, respectively. We also acknowledge the funding and general support received from the IITB-Monash Research Academy, DST, SERB and DBT India.

[1] W. A. Bickmore, Annu. Rev. Genomics Hum. Genet. 14, 67 (2013)

[2] B. D. Pope, T. Ryba, V. Dileep, F. Yue, W. Wu, O. Denas, D. L. Vera, Y. Wang, R. S. Hansen, T. K. Canfield, et al., Nature 515, 402 (2014).

[3] P.-C. Wei, C.-S. Lee, Z. Du, B. Schwer, Y. Zhang, J. Kao, J. Zurita, and F. W. Alt, PNAS 115, 1919 (2018).

[4] A. Sanyal, B. R. Lajoie, G. Jain, and J. Dekker, Nature 489, 109 (2012).

[5] E. Lieberman-Aiden, N. L. Van Berkum, L. Williams, M. Imakaev, T. Ragoczy, A. Telling, I. Amit, B. R. Lajoie, P. J. Sabo, M. O. Dorschner, et al., Science 326, 289 (2009).

[6] J. R. Dixon, S. Selvaraj, F. Yue, A. Kim, Y. Li, Y. Shen, M. Hu, J. S. Liu, and B. Ren, Nature 485, 376 (2012).

[7] E. P. Nora, B. R. Lajoie, E. G. Schulz, L. Giorgetti, I. Okamoto, N. Servant, T. Piolot, N. L. van Berkum, J. Meisig, J. Sedat, et al., Nature 485, 381 (2012).

[8] T. Nagano, Y. Lubling, C. Várnai, C. Dudley, W. Leung, Y. Baran, N. M. Cohen, S. Wingett, P. Fraser, and A. Tanay, Nature 547, 61 (2017).

[9] D. Baù, A. Sanyal, B. R. Lajoie, E. Capriotti, M. Byron, J. B. Lawrence, J. Dekker, and M. A. Marti-Renom, Nat. Struct. Mol. Biol. 18, 107 (2011).

[10] T. Nozaki, R. Imai, M. Tanbo, R. Nagashima, S. Tamura, T. Tani, Y. Joti, M. Tomita, K. Hibino, M. T. Kanemaki, et al., Mol. Cell 67, 282 (2017).

[11] B. Bintu, L. J. Mateo, J.-H. Su, N. A. SinnottArmstrong, M. Parker, S. Kinrot, K. Yamaya, A. N. Boettiger, and X. Zhuang, Science 362 (2018).

[12] Q. Szabo, A. Donjon, I. Jerković, G. L. Papadopoulos, T. Cheutin, B. Bonev, E. P. Nora, B. G. Bruneau, F. Bantignies, and G. Cavalli, Nat. Genet. 52, 1151 (2020).

[13] S. S. Rao, M. H. Huntley, N. C. Durand, E. K. Stamenova, I. D. Bochkov, J. T. Robinson, A. L. Sanborn, I. Machol, A. D. Omer, E. S. Lander, et al., Cell 159, 1665 (2014).

[14] M. J. Rowley and V. G. Corces, Nat. Rev. Genet. 19, 789 (2018).

[15] H. Belaghzal, T. Borrman, A. D. Stephens, D. L. Lafontaine, S. V. Venev, Z. Weng, J. F. Marko, and 
bioRxiv preprint doi: https://doi.org/10.1101/2021.02.17.431616; this version posted May 11, 2021. The copyright holder for this preprint

J. Dekker, Nat. Genet. pp. 1-12 (2021).

[16] E. Alipour and J. F. Marko, Nucleic Acids Res. 40, 11202 (2012).

[17] M. Mir, W. Bickmore, E. E. Furlong, and G. Narlikar, Development 146, dev182766 (2019).

[18] A. Goloborodko, J. F. Marko, and L. A. Mirny, Biophys. J. 110, 2162 (2016)

[19] G. Fudenberg, M. Imakaev, C. Lu, A. Goloborodko, N. Abdennur, and L. A. Mirny, Cell Rep. 15, 2038 (2016).

[20] A. Kaushal, G. Mohana, J. Dorier, I. Özdemir, A. Omer P. Cousin, A. Semenova, M. Taschner, O. Dergai, F. Marzetta, et al., Nat. Commun. 12, 1 (2021).

[21] S. J. Nair, L. Yang, D. Meluzzi, S. Oh, F. Yang, M. J. Friedman, S. Wang, T. Suter, I. Alshareedah, A. Gamliel, et al., Nat. Struct. Mol. Biol. 26, 193 (2019).

[22] D. Hnisz, K. Shrinivas, R. A. Young, A. K. Chakraborty, and P. A. Sharp, Cell 169, 13 (2017).

[23] N. S. Benabdallah, I. Williamson, R. S. Illingworth, L. Kane, S. Boyle, D. Sengupta, G. R. Grimes, P. Therizols, and W. A. Bickmore, Mol. Cell 76, 473 (2019).

[24] B. A. Gibson, L. K. Doolittle, M. W. Schneider, L. E. Jensen, N. Gamarra, L. Henry, D. W. Gerlich, S. Redding, and M. K. Rosen, Cell 179, 470 (2019).

[25] K. Shrinivas, B. R. Sabari, E. L. Coffey, I. A. Klein, A. Boija, A. V. Zamudio, J. Schuijers, N. M. Hannett, P. A. Sharp, R. A. Young, et al., Mol. Cell 75, 549 (2019).

[26] Y. Zhang, R. P. McCord, Y.-J. Ho, B. R. Lajoie, D. G. Hildebrand, A. C. Simon, M. S. Becker, F. W. Alt, and J. Dekker, Cell 148, 908 (2012).

[27] D. Jost, P. Carrivain, G. Cavalli, and C. Vaillant, Nucleic Acids Res. 42, 9553 (2014).

[28] J. J. Parmar and R. Padinhateeri, Curr. Opin. Struct. Biol. 64, 111 (2020).

[29] K. Kumari, B. Duenweg, R. Padinhateeri, and J. R. Prakash, Biophys. J. 118, 2193 (2020).

[30] L. Giorgetti, R. Galupa, E. P. Nora, T. Piolot, F. Lam, J. Dekker, G. Tiana, and E. Heard, Cell 157, 950 (2014).

[31] S. Bianco, D. G. Lupiáñez, A. M. Chiariello, C. Annunziatella, K. Kraft, R. Schöpflin, L. Wittler, G. Andrey, M. Vingron, A. Pombo, et al., Nat. Genet. 50, 662 (2018).

[32] G. D. Bascom, C. G. Myers, and T. Schlick, PNAS 116, 4955 (2019)

[33] M. Di Pierro, B. Zhang, E. L. Aiden, P. G. Wolynes, and J. N. Onuchic, PNAS 113, 12168 (2016).

[34] A. Rosa and R. Everaers, PLoS Comput. Biol. 4, e1000153 (2008)

[35] M. Di Stefano, J. Paulsen, D. Jost, and M. A. MartiRenom, Curr. Opin. Genet. Dev. 67, 25 (2021).

[36] C. T. Clarkson, E. A. Deeks, R. Samarista, H. Mamayusupova, V. B. Zhurkin, and V. B. Teif, Nucleic Acids Res. 47, 11181 (2019).

[37] M. Conte, L. Fiorillo, S. Bianco, A. M. Chiariello, A. Esposito, and M. Nicodemi, Nat. Commun. 11, 1 (2020).

[38] Y. Qi and B. Zhang, PLoS Comput. Biol. 15, e1007024 (2019).

[39] G. Shi, L. Liu, C. Hyeon, and D. Thirumalai, Nat. Commun. 9, 1 (2018).

[40] Q. MacPherson, B. Beltran, and A. J. Spakowitz, PNAS 115, 12739 (2018).

[41] G. Bajpai and R. Padinhateeri, Biophys. J. 118, 207
(2020).

[42] C. A. Brackey, D. Marenduzzo, and N. Gilbert, Nature Methods 17, 767 (2020).

[43] R. Bird, C. Curtiss, R. Armstrong, and O. Hassager, Dynamics of polymeric liquids, kinetic theory (volume 2 ) (1987).

[44] T. Soddemann, B. Dünweg, and K. Kremer, Eur. Phys. J. E 6, 409 (2001).

[45] A. Santra, K. Kumari, R. Padinhateeri, B. Dünweg, and J. R. Prakash, Soft Matter 15, 7876 (2019).

[46] J. Des Cloizeaux, J. Phys 41, 223 (1980).

[47] R. Imai, T. Nozaki, T. Tani, K. Kaizu, K. Hibino, S. Ide, S. Tamura, K. Takahashi, M. Shribak, and K. Maeshima, Mol. Biol. Cell 28, 3349 (2017).

[48] K. Maeshima, S. Tamura, J. C. Hansen, and Y. Itoh, Curr. Opin. Cell Biol. 64, 77 (2020).

[49] H. Strickfaden, T. O. Tolsma, A. Sharma, D. A. Underhill, J. C. Hansen, and M. J. Hendzel, Cell (2020).

[50] T. T. Pham, M. Bajaj, and J. R. Prakash, Soft Matter 4, 1196 (2008).

[51] T. T. Pham, B. Duenweg, and J. R. Prakash, Macromolecules 43, 10084 (2010).

[52] T. Germier, S. Kocanova, N. Walther, A. Bancaud, H. A. Shaban, H. Sellou, A. Z. Politi, J. Ellenberg, F. Gallardo, and K. Bystricky, Biophys. J. 113, 1383 (2017).

[53] A. Amitai and D. Holcman, Physical Review E 97, 032417 (2018)

[54] Y. Zhang and O. K. Dudko, Annual review of biophysics 45, 117 (2016).

[55] N. M. Toan, G. Morrison, C. Hyeon, and D. Thirumalai, J. Phys. Chem. B 112, 6094 (2008).

[56] J. G. Yang, T. S. Madrid, E. Sevastopoulos, and G. J. Narlikar, Nat. Struct. Mol. Biol. 13, 1078 (2006).

[57] S. H. Sandholtz, Q. MacPherson, and A. J. Spakowitz, PNAS 117, 20423 (2020).

[58] C. A. Brackley, J. M. Brown, D. Waithe, C. Babbs, J. Davies, J. R. Hughes, V. J. Buckle, and D. Marenduzzo, Genome biology 17, 1 (2016).

[59] N. Khanna, Y. Zhang, J. S. Lucas, O. K. Dudko, and C. Murre, Nature communications 10, 1 (2019).

[60] G. Shi and D. Thirumalai, Physical Review X 11, 011051 (2021).

[61] E. Delbarre, K. Ivanauskiene, J. Spirkoski, A. Shah, K. Vekterud, J. Ø. Moskaug, S. O. Bøe, L. H. Wong, T. Küntziger, and P. Collas, Genome research 27, 913 (2017).

[62] J. R. Prakash, Korea-Aust. Rheol. J. 21, 245 (2009).

[63] J. R. Prakash, Curr. Opin. Colloid Interface Sci. 43, 63 (2019).

[64] R. Prabhakar, C. Sasmal, D. A. Nguyen, T. Sridhar, and J. R. Prakash, Phys. Rev. Fluids 2, 011301 (2017).

[65] R. Prabhakar and J. R. Prakash, J. Non-Newtonian Fluid Mech. 116, 163 (2004).

[66] C. M. Schroeder, J. Rheol. 62, 371 (2018).

[67] P. Sunthar and J. R. Prakash, Europhys. Lett. 75, 77 (2006).

[68] P. Sunthar and J. R. Prakash, Macromolecules 38, 617 (2005).

[69] C. M. Schroeder, E. S. Shaqfeh, and S. Chu, Macromolecules 37, 9242 (2004). 